\title{
AW Mic: The RR Lyrae That Wasn't
}

\author{
GERRI BERnARD ${ }^{1, *}$ \\ ${ }^{1}$ Brisbane Girls Grammar School, Brisbane, QLD, Australia \\ "Corresponding author: gbernard@bggs.qld.edu.au
}

\begin{abstract}
AW Mic is a neglected star that has previously been categorised as an 'unusual' RRc variable. This paper makes use of new and archival observations of AW Mic to establish that this star is no longer varying in magnitude and should no longer be classified as an RR Lyrae variable. Mid magnitudes of $9.171 \pm 0.026$ and $9.001 \pm 0.014$ are reported for this star in the $B$ - and $V$-bands, respectively. (a)(1) $\odot \odot 2021$ Astronomy Theory, Observations and Methods Journal
\end{abstract}

Keywords: $\quad$ RR Lyrae, Variable stars

https://doi.org/10.32374/atom.2020.2.2

\section{INTRODUCTION}

RR Lyrae are low mass, evolved stars that have moved off the main sequence and initiated core helium burning. These stars can radially pulsate in various modes (fundamental, first overtone, or both) and generally exhibit periods of $0.2-1$ days. They are found at the intersection of the horizontal branch and the classical instability strip (Catelan \& Smith, 2015).

Stars on the horizontal branch may cross the instability strip in either direction, resulting in an increase in observed period for those stars evolving from blue to red, or a decrease in period for those stars evolving from red to blue. RR Lyrae that have moved out of the instability strip in either direction would cease pulsation and become either blue HB stars or red AGB stars (Le Borgne et al., 2007).

First classified as a horizontal branch star by MacConnell, Frye, Bidelman and Bond (1971), AW Mic (HD 202759) was later found by Przybylski and Bessell (1974) to be an unusual RRc with a 'complex and irregular light curve'. In their paper, Przbylski and Bessell present a reasonable, low amplitude light curve ( $\Delta$ magnitude $<0.1$ ) based on 164 observations and generate a period estimate of 11.5 hours for this star, but note that more observations would be required to deduce a full description of the star's behaviour. They cite the relatively high effective temperature of the star $(7400 \mathrm{~K})$ as evidence that this star may lie close to the blue edge of the instability strip.

Some authors have concurred with Przybylski and Bessel's assessment that this is an RR Lyrae star based on their own measurements of $T_{\text {eff }}$ (Kodaira \& Philip, 1984), however, more recent sources find that the star is more similar to a non-varying blue horizontal branch star (Kinman et al., 2000). A summary of some of the reported properties of AW Mic is presented in Table 1. Overall, the period appears not to be well-established and the amplitude of the magnitude in the V-band is underreported. The effective temperature and metallicity of AW Mic seem to be corroborated across various sources.

\section{METHODS}

\section{Observations}

The data for this project was gathered using the The Dorothy Hill Observatory (DHO), located at the Mar- 
Table 1. Reported properties of AW Mic

\begin{tabular}{|c|c|c|}
\hline Property & Value & Source \\
\hline ICRSd Right Ascension & 319.775 & (Gaia et al., 2018) \\
\hline ICRSd Declination & -33.919 & \\
\hline \multirow[t]{3}{*}{ Period } & $0.478 \mathrm{~d}(11.5 \mathrm{hrs})$ & (Przybylski \& Bessell, 1974) \\
\hline & $0.478 \mathrm{~d}(11.5 \mathrm{hrs})$ & (Samus', Kazarovets, Durlevich, Kireeva, \& Pastukhova, 2017) \\
\hline & $0.305684 \mathrm{~d}(7.3664 \mathrm{hrs})$ & (Kafka, 2016) \\
\hline \multirow[t]{5}{*}{$\mathrm{V}_{\text {mag }}$} & $8.86^{a}$ & (Jayasinghe et al., 2019; Shappee et al., 2014) \\
\hline & $8.97^{b}$ & (Przybylski \& Bessell, 1974) \\
\hline & 9.015 & (Henden et al., 2016) \\
\hline & $9.09^{c}$ & (Kinman et al., 2000) \\
\hline & $9.04-9.13$ & (Kafka, 2016) \\
\hline \multirow[t]{2}{*}{$\operatorname{Max} V_{m a g}$-Min $V_{m a g}$} & 0.08 & (Przybylski \& Bessell, 1974) \\
\hline & 0.09 & (Kafka, 2016) \\
\hline \multirow[t]{4}{*}{$\mathrm{T}_{e f f}$} & $7400 \mathrm{~K}$ & (Kodaira \& Philip, 1984) \\
\hline & $7431 \mathrm{~K}$ & (Wilhelm, Beers, \& Gray, 1999) \\
\hline & $7465 \mathrm{~K}$ & (Kinman et al., 2000) \\
\hline & $7500 \mathrm{~K}$ & (Gaia et al., 2018) \\
\hline \multirow[t]{3}{*}[\mathrm{Fe}/\mathrm{H}]{} & -2.35 & (Cortés et al., 2009) \\
\hline & -2.37 & (Wilhelm et al., 1999) \\
\hline & -2.40 & (Kinman et al., 2000) \\
\hline
\end{tabular}


rapatta Memorial Outdoor Education Centre, near Imbil, QLD, Australia. $B$ - and $V$-band observations of AW Mic were taken between 3 August 2020-7 August 2020 with an FLI CCD attached to a roboticallycontrolled 356mm Planewave reflecting telescope. The pixel scale of the camera was 0.72 "/pixel in $1 \mathrm{x} 1$ binning mode with a 50' x 50' field of view. Exposure time was ten seconds for all of the 328 observations, which resulted in an approximate integrated ADU count value of 250,000 from the target star, and a cadence of five minutes was used.

\section{Photometry}

The images and photometry were processed using SEK (Source Extractor Kron) (Bertin \& Arnouts, 1996) and PSX (PSFEx) (Bertin, 2011) files from the Our SolarSiblings Pipeline (M. Fitzgerald, 2018). Data analysis was carried out using astrosource (M. T. Fitzgerald, Gomez, Salimpour, Singleton, \& Wibowo, 2021), a python script that carries out differential photometry based on optimal comparison stars in each filter. Astrosource identified the comparison stars shown in Figure 1 as being the least variable in the field (having the smallest standard deviation of differential magnitude across all images used), with variabilities between 0.004 and 0.03 for all comparison stars in both filters. Due to a lack of stars in the field with sufficient brightness in the $B$-band, only one suitable comparison star was identified by astrosource for comparison in this band. Differential photometry was then performed using known comparison star magnitudes from APASS (Henden et al., 2016) and calibrated magnitudes for AW Mic for each observation were generated in $B$ - and $V$-bands.

\section{Spectroscopy}

Spectroscopy was carried out using the FLOYDS spectrograph on the Las Cumbres Observatory $2.0 \mathrm{~m}$ telescope at Siding Springs, Australia (Brown et al., 2013). FLOYDS is a cross-dispersed, low resolution ( $R$ 550) spectrograph with wavelength coverage of $3200 \AA-10000 \AA$. Two highly similar spectra were measured on HJD 2459108.85924 and HJD 2459108.85925 .

\section{RESULTS AND DISCUSSION}

\section{Photometry}

Table 2 shows photometric results for DHO observations of AW Mic, including calibrated magnitudes in

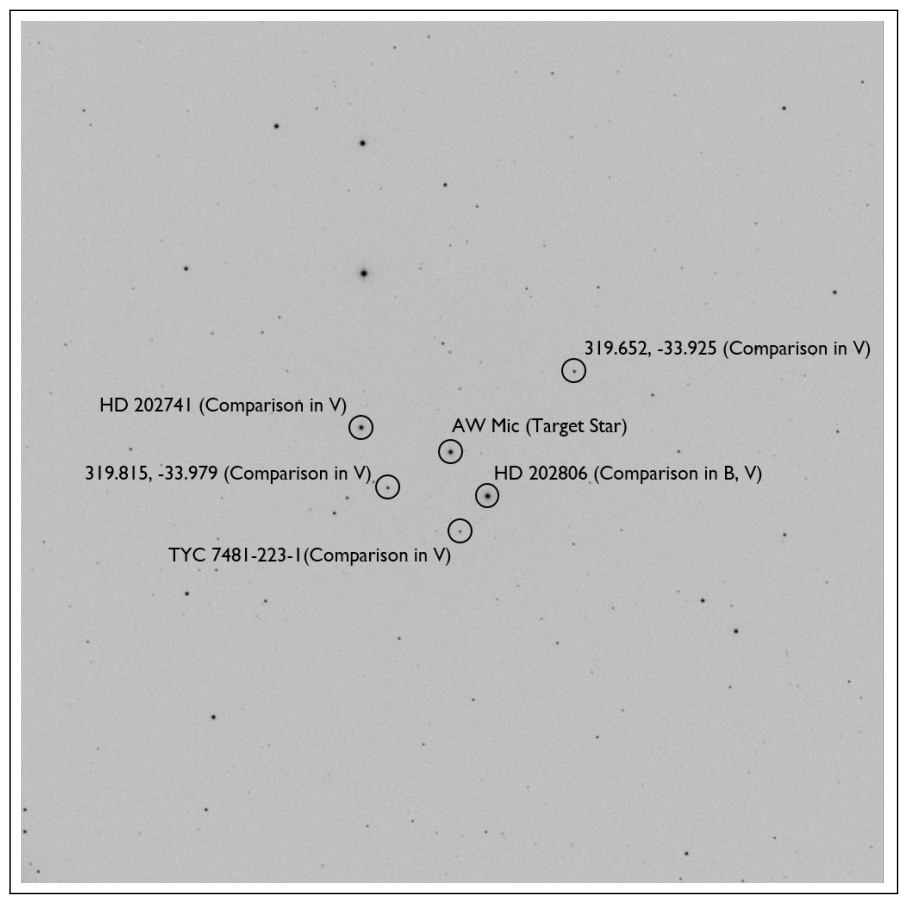

Fig. 1. Inverted image of field analysed in DHO observations. Field of view is 50' x 50'.

$B$ - and $V$-bands. $V$-band magnitudes match well with those previously reported in the literature.

Figure 2 shows a plot of period likelihood vs tested periods, as generated by the astrosource analysis. The possible expected periods of AW Mic from Table 1 are shown to have no more likelihood than any other period in the expected range of an RR Lyrae variable.

Figures 3 and 4 show calibrated magnitudes of AW Mic in $B$ - and $V$-bands, generated from photometry derived from 328 DHO observations (see Table 2). There is quite a bit of scatter and attempts to fold the lightcurve around previously reported periods for AW Mic resulted in plots that showed no evidence of pulsation.

Photometry results from observations at the DHO were compared to photometry data from the All Sky Automated Survey (Pojmanski \& Maciejewski, 2005) and the Wide Angle Search for Planets (Butters et al., 2010). The ASAS data is based on observations made between HJD 2451872.53857-2455166.56762 and the SuperWASP data is from observations made between HJD 2453862.551-2454614.69.

Figures 5 and 6 show calibrated, phased light curves from SuperWASP and ASAS, respectively, for comparison to those generated from DHO observations. Both comparison light curves were generated assuming the AAVSO reported period of $0.3056840 \mathrm{~d}$ 
Table 2. Observation results from DHO.

\begin{tabular}{cc}
\hline B-Band & \\
\hline First Observation BJD & 2459072.108 \\
Last Observation BJD & 2459078.271 \\
Minimum Magnitude & $9.218 \pm 0.026$ \\
Mid Magnitude & $9.171 \pm 0.026$ \\
Maximum Magnitude & $9.124 \pm 0.026$ \\
\hline V-Band & \\
\hline First Observation BJD & 2459072.086 \\
Last Observation BJD & 2459078.275 \\
Minimum Magnitude & $8.900 \pm 0.014$ \\
Mid Magnitude & $9.001 \pm 0.014$ \\
Maximum Magnitude & $9.101 \pm 0.014$ \\
\hline
\end{tabular}

for AW Mic. As with the results from DHO observations, no periodicity is evident for the expected period.

As an additional test, phased SuperWASP and ASAS light curves were generated using Przybylski and Bessel's (1974) reported period of 0.478d. These are shown in Figures 7 and 8 and, again, no periodicity is evident.

This leads to the conclusion that AW Mic, which may have been variable in the past, is not currently changing magnitude in a periodic way and certainly not in a manner consistent with the behaviour of an RRc. Therefore, this star should no longer be classified as an RRc variable.

\section{Spectroscopy}

Figure 9 shows a spectrum of AW Mic between $3300 \AA$ $10500 \AA$. Visual inspection indicates that the metallicities reported in the literature for AW Mic seem reasonable (see Table 1 ).

\section{CONCLUSION}

This research demonstrated that AW Mic, previously assumed to be an RRc variable star, appears to be non-variable. Recent observations from the DHO that demonstrate non-variability are confirmed by archival observations made by the All Sky Automated Survey and by the Wide Angle Search for Planets, indicating that AW Mic has been non-variable for at least twenty years. Therefore, it is likely that AW Mic will need to be removed from common databases and catalogues for variable stars.

\section{FUNDING}

Observations in this paper made use of telescopes at the Dorothy Hill Observatory (DHO) in Queensland, Australia. The DHO is a facility established and funded by Brisbane Girls Grammar School.

\section{ACKNOWLEDGMENTS}

The author would like to acknowledge Dr Michael Fitzgerald, Dr David Trappett, and Anthony Lumsden for their major contributions to the data collection for this project. This paper makes use of data from the DR1 of the WASP data (Butters et al. 2010) as provided by the WASP consortium, and the computing and storage facilities at the CERIT Scientific Cloud, reg. no. CZ.1.05/3.2.00/08.0144 which is operated by Masaryk University, Czech Republic. This research has made use of the International Variable Star Index (VSX) database, operated at AAVSO, Cambridge, Massachusetts, USA. This research has made use of the SIMBAD database, operated at CDS, Strasbourg, France.

\section{SUPPLEMENTAL DOCUMENTS REFERENCES}

Bertin, E. (2011). Automated morphometry with sextractor and psfex. In Astronomical data analysis software and systems $x x$ (Vol. 442, p. 435).

Bertin, E., \& Arnouts, S. (1996). Sextractor: Software for source extraction. Astronomy and Astrophysics Supplement Series, 117(2), 393-404.

Brown, T., Baliber, N., Bianco, F., Bowman, M., Burleson, B., Conway, P., ... et al. (2013). Las cumbres observatory global telescope network. Publications of the Astronomical Society of the Pacific, 125(931), 1031.

Butters, O., West, R., Anderson, D., Cameron, A., Clarkson, W., Enoch, B., ... Todd, I. (2010, 09). The first wasp public data release. $h t t p: / / d x$.doi.org/10.1051/00046361/201015655, 520. doi:

Catelan, M., \& Smith, H. A. (2015). Pulsating stars (1st ed.). Weinheim: Wiley-VCH Verlag GmbH Co. KGaA. 


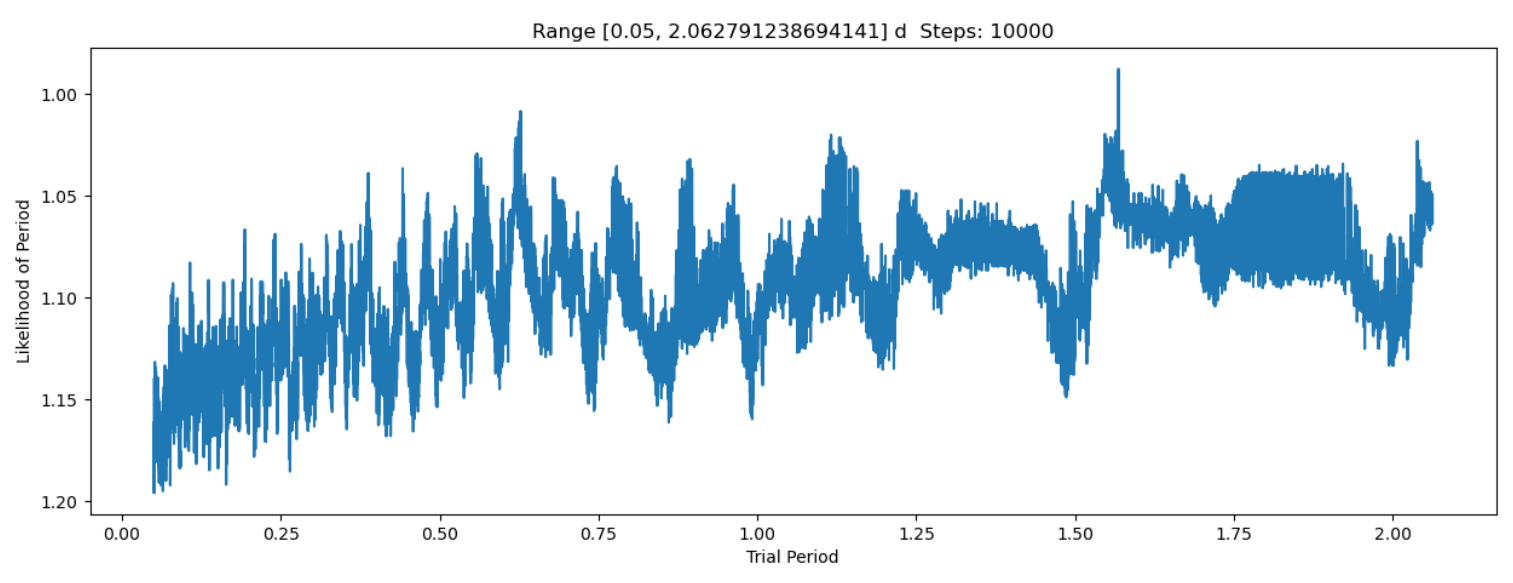

Fig. 2. Phase dispersion minimization (PDM) likelihood plot in $V$-band from DHO observations. Note that the $\mathrm{y}$-axis (unitless) is inverted, with larger numbers indicating higher likelihood of particular periods (in days), as determined by PDM analysis.

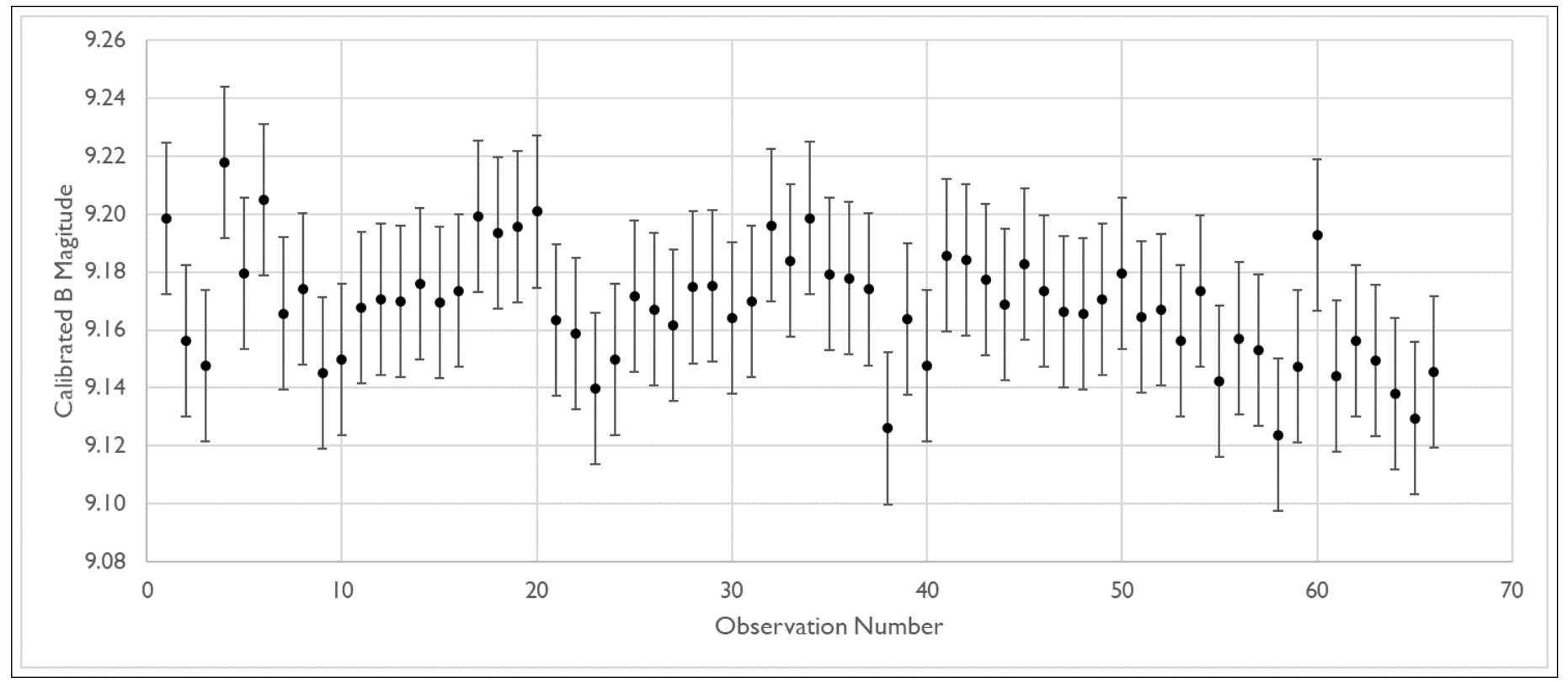

Fig. 3. Calibrated $B$-band magnitude for AW Mic from $66 \mathrm{DHO}$ observations. The BJDs of the first and last observation shown are 2459072.10775511 and 2459078.27149761, respectively. 




Fig. 4. Calibrated V-band magnitude for AW Mic from 262 DHO observations. The BJDs of the first and last observation shown are 2459072.08644584 and 2459078.27481956, respectively.

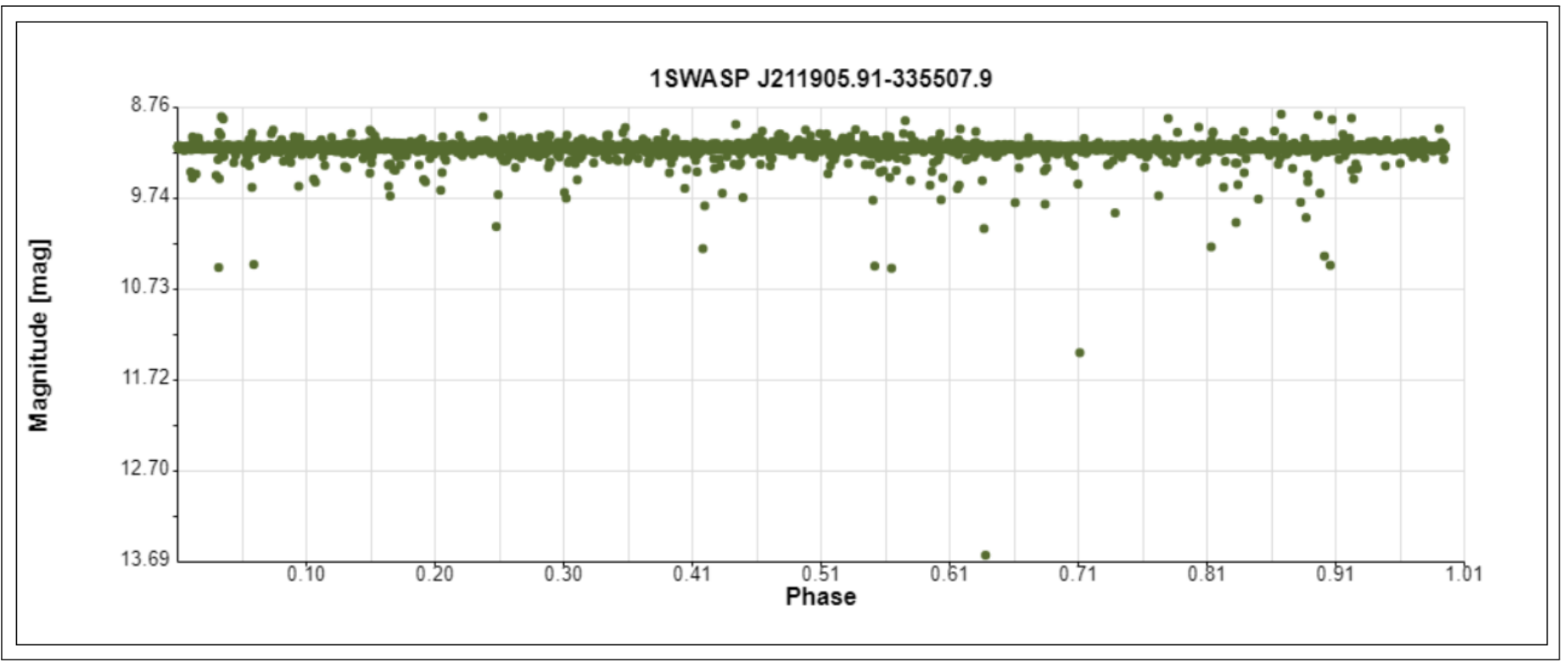

Fig. 5. Calibrated, phased light curve from SUPERWasp observations of RA: 319.77465/DEC: -33.918869, using AAVSO reported period of 0.3056840d (Butters et al., 2010), accessed on 12 October 2020. 


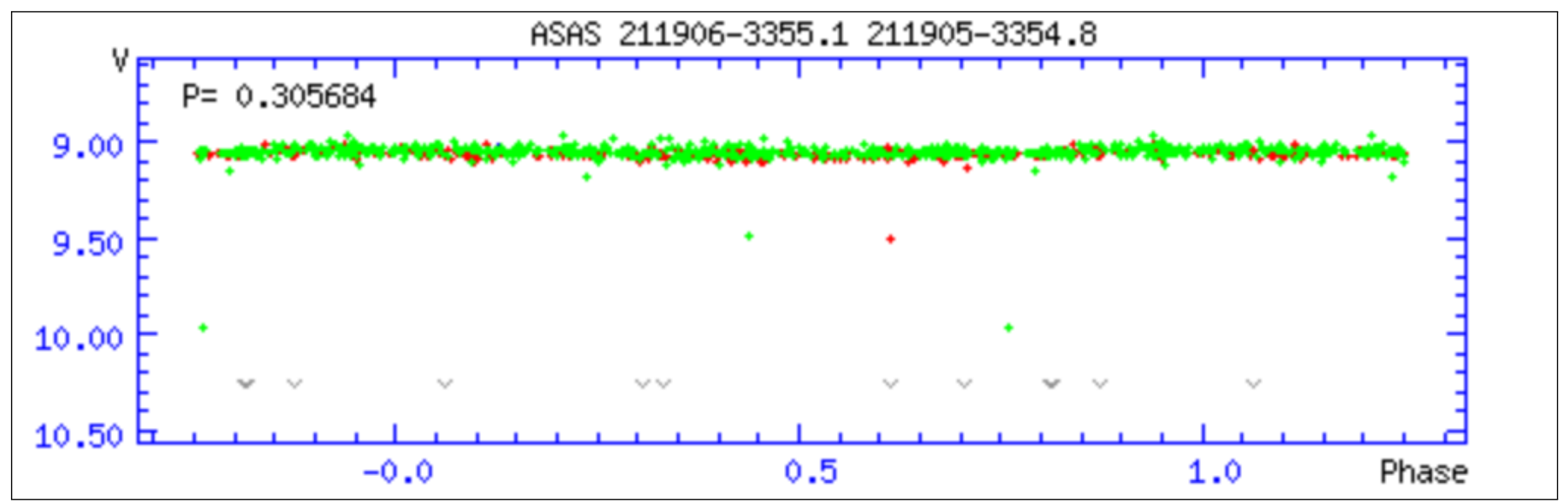

Fig. 6. Calibrated, phased light curve in $V$-band from ASAS observations, using AAVSO reported period of 0.305684d (Pojmanski \& Maciejewski, 2005), accessed on 12 October 2020. Y-axis is calibrated magnitude.

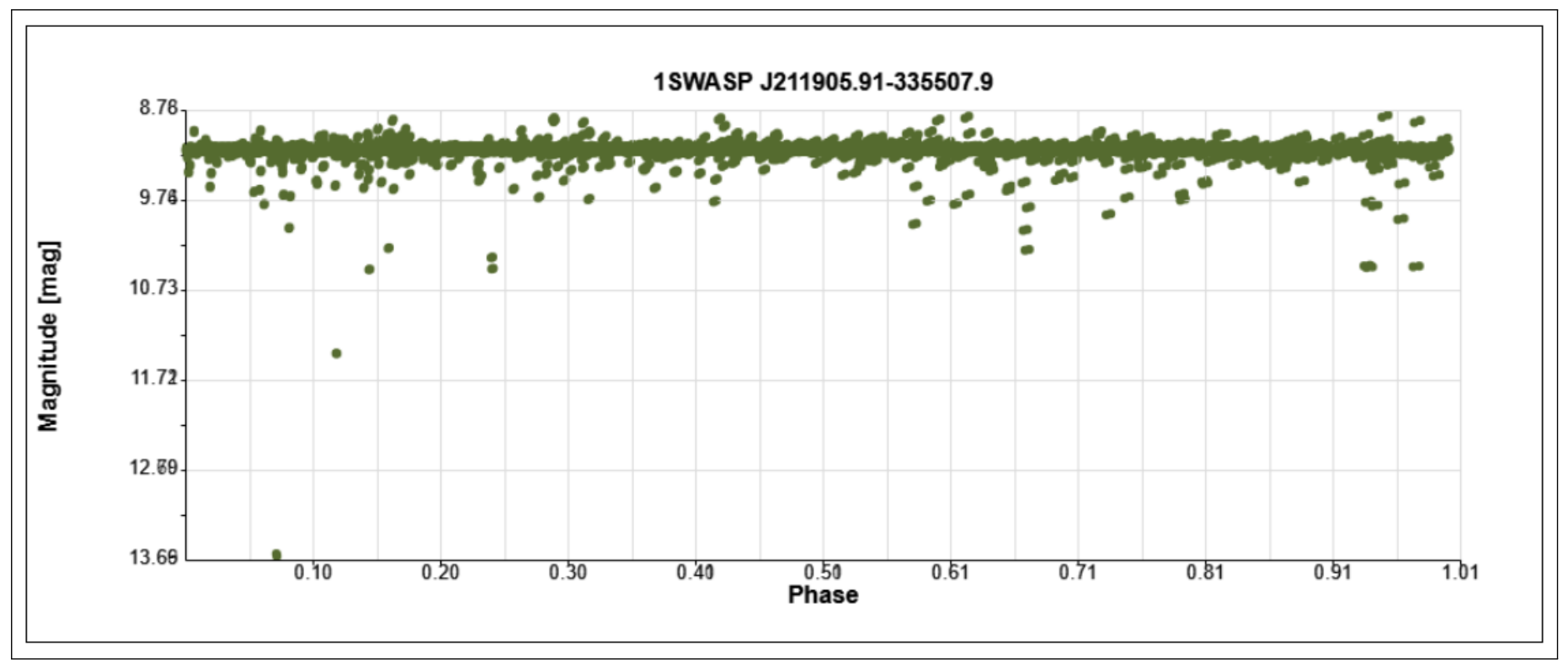

Fig. 7. Calibrated, phased light curve from SUPERWasp observations of RA: 319.77465/DEC: -33.918869 (Butters et al., 2010), using Przybylski and Bessel's (1974) reported period of 0.478d, accessed on 12 October 2020. 


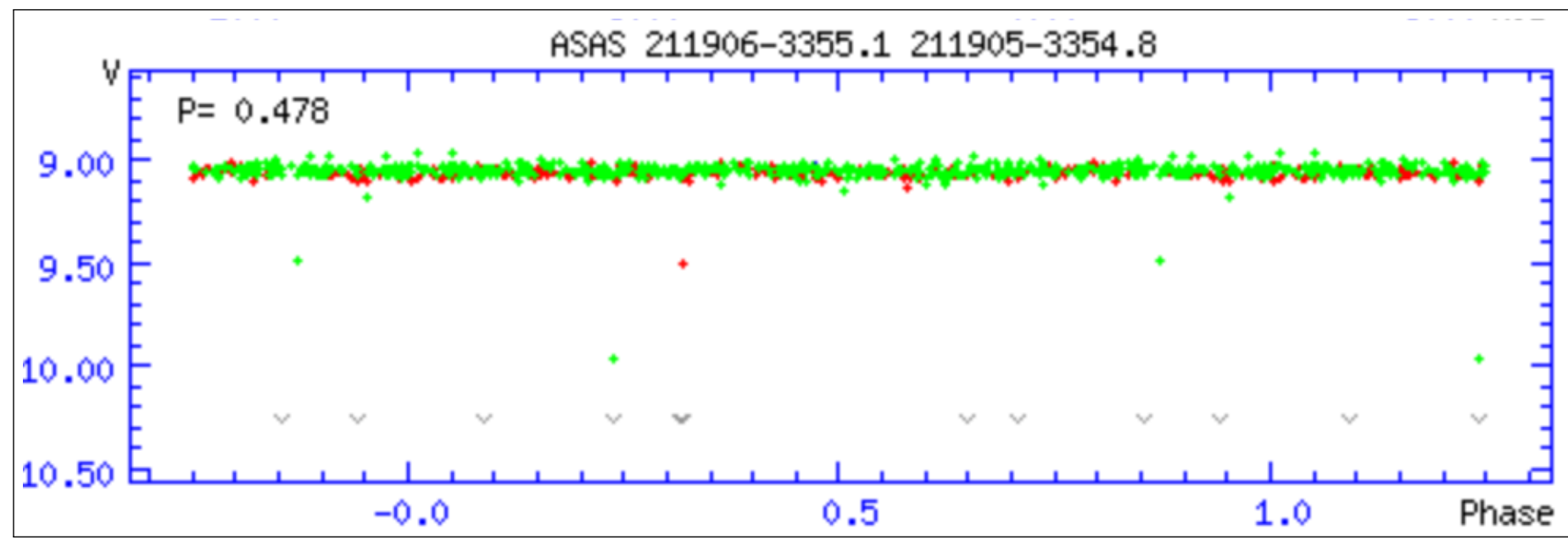

Fig. 8. Calibrated, phased light curve in V-band from ASAS observations (Pojmanski \& Maciejewski, 2005), using Przybylski and Bessel's (1974) reported period of 0.478d, accessed on 12 October 2020. Y-axis is calibrated magnitude.

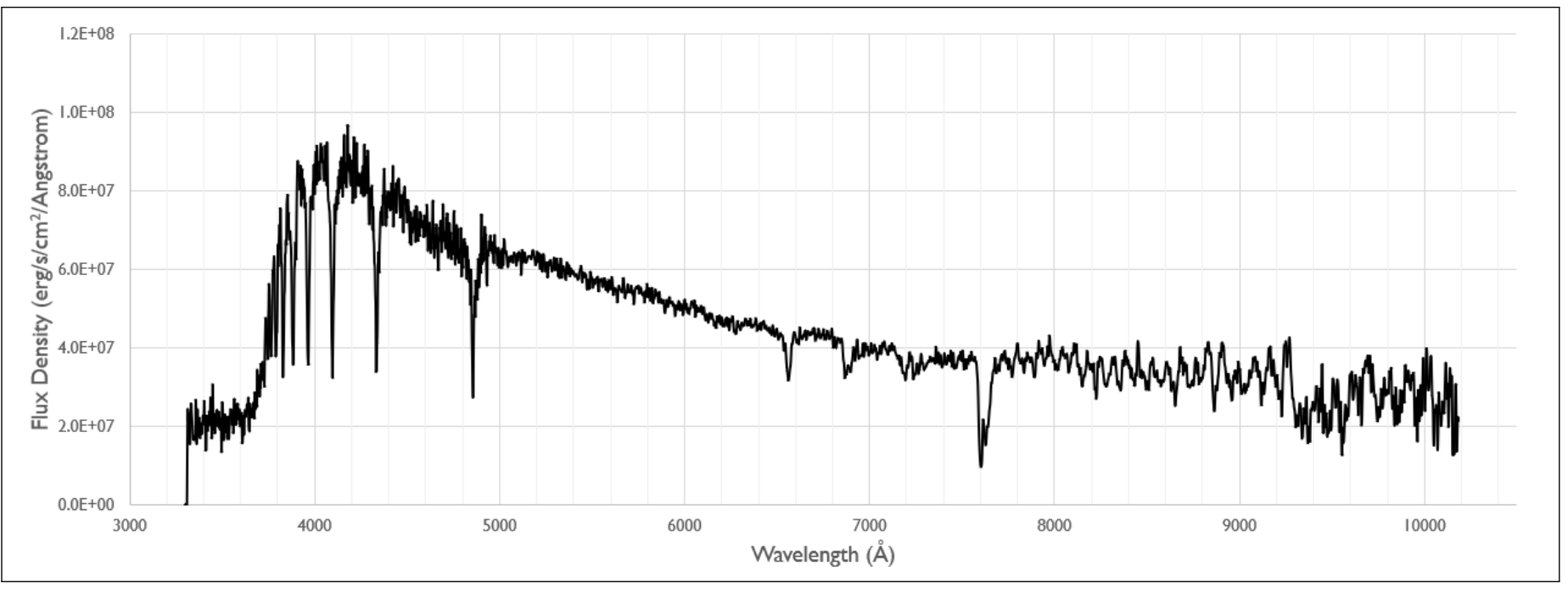

Fig. 9. FLOYDS spectrum of AW Mic from $3300 \AA$ to $10100 \AA$. 
Cortés, C., Silva, J., Recio-Blanco, A., Catelan, M., Do Nascimento Jr, J., \& De Medeiros, J. (2009). An overview of the rotational behavior of metalpoor stars. The Astrophysical Journal, 704(1), 750.

Fitzgerald, M. (2018). The our solar siblings pipeline: Tackling the data issues of the scaling problem for robotic telescope based astronomy education projects.

Fitzgerald, M. T., Gomez, E., Salimpour, S., Singleton, J., \& Wibowo, R. W. (2021). "astrosource": automating optical astronomy measurement, calibration and analysis for variable stellar sources from provided photometry. Journal of Open Source Software, 6(58), 2641.

Gaia, C., Brown, A., Vallenari, A., Prusti, T., de Bruijne, J., Babusiaux, C., ... others (2018). Gaia data release 2 summary of the contents and survey properties. Astronomy \& Astrophysics, 616(1).

Henden, A. A., Templeton, M., Terrell, D., Smith, T. C., Levine, S., \& Welch, D. (2016, January). VizieR Online Data Catalog: AAVSO Photometric All Sky Survey (APASS) DR9 (Henden+, 2016). VizieR Online Data Catalog, II/336.

Jayasinghe, T., Stanek, K., Kochanek, C., Shappee, B., Holoien, T. W., Thompson, T. A., ... others (2019). The asas-sn catalogue of variable starsii. uniform classification of 412000 known variables. Monthly Notices of the Royal Astronomical Society, 486(2), 1907-1943.

Kafka, S. (2016). Observations from the aavso international database.

Kinman, T., Castelli, F., Cacciari, C., Bragaglia, A., Harmer, D., \& Valdes, F. (2000). A spectroscopic study of field bhb star candidates. arXiv preprint astro-ph/0006179.

Kodaira, K., \& Philip, A. (1984). High-dispersion spectroscopic investigation of field horizontalbranch, high-luminosity, and main-sequence stars. The Astrophysical Journal, 278, 208-214.

Le Borgne, J., Paschke, A., Vandenbroere, J., Poretti, E., Klotz, A., Boër, M., ... Acerbi, F. (2007). Stellar evolution through the ages: period variations in galactic rrab stars as derived from the geos database and tarot telescopes. Astronomy \& Astrophysics, 476(1), 307-316.

MacConnell, D., Frye, R., Bidelman, W., \& Bond, H. E. (1971). Discoveries on southern objective-prism plates. ii. new probable field horizontal-branch stars. Publications of the Astronomical Society of the Pacific, 83(491), 98.

Pojmanski, G., \& Maciejewski, G. (2005). The all sky automated survey. catalog of variable stars. iv. $18^{\wedge} \mathrm{h}-24^{\wedge} \mathrm{h}$ quarter of the southern hemisphere. Acta Astronomica, 55, 97-122.

Przybylski, A., \& Bessell, M. (1974). An unusual rr lyrae-type variable. Publications of the Astronomical Society of the Pacific, 86(512), 403.

Samus', N., Kazarovets, E., Durlevich, O., Kireeva, N., \& Pastukhova, E. (2017, 01). General catalogue of variable stars: Version gcvs 5.1. Astronomy Reports, 61, 80-88. doi:

Shappee, B. J., Prieto, J., Grupe, D., Kochanek, C., Stanek, K., De Rosa, G., ... others (2014). The man behind the curtain: X-rays drive the uv through nir variability in the 2013 active galactic nucleus outburst in ngc 2617. The Astrophysical Journal, 788(1), 48.

The Dorothy Hill Observatory. (n.d.). Retrieved from https://www.bggs.qld.edu.au/about -brisbane-girls-grammar/facilities/ dorothy-hill-observatory/

Wilhelm, R., Beers, T. C., \& Gray, R. O. (1999). Spectroscopy of hot stars in the galactic halo. ii. the identification and classification of horizontalbranch and other a-type stars. The Astronomical Journal, 117(5), 2308. 